\title{
Colecho en el hogar, lactancia materna y. muerte súbita del lactante. Recomendaciones para los profesionales de la salud
}

\author{
Bedsharing at home, breastfeeding and sudden infant death. \\ Recommendations for health professionals
}

\author{
Subcomisión de Lactancia Materna y Grupo de Trabajo de Muerte Súbita e Inesperada \\ del Lactante
}

Resumen publicado en Arch Argent Pediatr 2017;115(5):520.

\section{RESUMEN}

El Grupo de Trabajo en Muerte Súbita e Inesperadadel Lactante, junto conlaSubcomisión deLactancia Materna delaSociedad Argentina de Pediatría, elaboraron nuevas recomendaciones sobre la práctica del colecho, en la cual el niño duerme en la misma superficie junto a su madre, situación que es motivo de controversia.

El colecho favorece la lactancia materna, que, a su vez, es protectora de la muerte súbita del lactante. Un pequeño grupo de niños presenta mayor riesgo de muerte súbita del lactante $\mathrm{y}$ accidentes fatales durante el colecho en ciertas circunstancias, que incluyen dormir en un sillón o sofá, padres fumadores, ingesta de sedantes, drogas y/o consumo de alcohol, niños prematuros y/o de bajo peso. El colecho en niños alimentados con leche humana, sin los factores de riesgo mencionados y con padres responsables de implementar un ambiente de sueño seguro, no aumenta el riesgo de muerte súbita del lactante. Esta guía no recomienda taxativamente la prohibición del colecho. Instruye a los profesionales dela salud a propalar a las familias un mensaje balanceado que incluya tanto los riesgos como los beneficios del colecho, lo que les permite a los padres una decisión informada al respecto. El documento señala que la cohabitación sin colecho es el lugar más seguro para los bebés al momento de dormir.

Palabras clave: muerte súbita del lactante, lactancia materna, colecho, cohabitación.

\begin{abstract}
The Sudden and Unexpected Infant Death Task Force together with the Subcommittee on Breastfeeding of the Sociedad Argentina de Pediatría have issued updated recommendations on bedsharing with the parents, a practice which remains controversial.

Sleeping with themothermaximizesbreastfeeding, which is protective against Sudden Infant Death. There is a small group of infants that have been associated with an increased risk of Sudden Infant Death and fatal sleeping accidents in certain circumstances. These hazards include parental smoking, sedating drugs or medication and alcohol consumption prior to sleep and sofasharing situations. Bedsharing by breastfeeding mothers with their infants, in the absence of the above-mentioned risk factors, and with parents aware of how to ensure a safe infant sleep environment has not been shown to be associated with increased risk of Sudden Infant Death. This guidance does not advise on telling parents that they must never sleep with the baby, but rather instructs health professionals to give parents balanced advice to allow informed decision making, emphasizing the concept that parental room sharing without bedsharing is the safest place for the babies to sleep.

Key words: sudden infant death, breastfeeding, bedsharing, room-sharing.

http: / / dx.doi.org/10.5546/aap.2017.S105

Cómo citar: Subcomisión de Lactancia Materna y Grupo de Trabajo de Muerte Súbita e Inesperada del Lactante. Colecho en el hogar, lactancia materna y muerte súbita del lactante. Recomendaciones para los profesionales de la salud. Arch Argent Pediatr 2017;115 Supl 5:S105-S110.
\end{abstract}

Correspondencia: alejandro.jenik@ hospitalitaliano.org.ar

Dra. Roxana Conti: roxanaconti@hotmail. com

Financiamiento:

Ninguno.

Conflicto de intereses: Ninguno que declarar.

Recibido: 20-5-2017 Aceptado: 22-5-2017
Coordinadores: Dra. Roxana G. Conti y Dr. Alejandro Jenik A. ${ }^{\mathrm{b}}$

a. Subcomisión de Lactancia Materna de la Sociedad Argentina de Pediatría: Dra. Roxana Conti, Dra. Mónica Waisman, Dr. Antonio Morilla, Dra. Vera May, Dra. Adriana Acquavita, Lic. Mónica Tesone, Lic. Cristina Malerba, Dra. Rosa Berenstein, Dra. Elda Ibañez, Dra. Patricia Barrios Skrok, Dr. Gustavo Hugo Sager, Lic. Rosa del Valle Herrera, Lic. Viviana Pereira, Lic. Graciela Sánchez.

b. Grupo de Trabajo de Muerte Súbita e Inesperada del Lactante de la Sociedad Argentina de Pediatría: Dr. Alejandro Jenik, Dra. Estela Grad, Dra. Letizia Fazola, Dr. Manuel Rocca Rivarola, Dra. Norma Rossato, Dra. Virginia Orazi.

Consultoras: Dra. María Luisa Ageitos y Dra. Norma Rossato 


\section{INTRODUCCIÓN}

El lugar más seguro para que duerma el bebé es en la habitación de los padres, en una cuna ubicada junto a su cama..$^{1-3}$ Sin embargo, el colecho constituye una práctica ancestral ${ }^{4-6}$ de la humanidad que tiene una fuerte asociación positiva con la lactancia ${ }^{7-10}$ y se extiende a diferentes ámbitos: hogares, ${ }^{11}$ hospitales ${ }^{12} \mathrm{y}$ laboratorios de investigación. ${ }^{10}$

Se define "colecho" a la circunstancia en la cual el niño duerme en la misma cama junto a su madre $\mathrm{u}$ otros miembros de la familia, todas las noches, durante, al menos, cuatro horas. ${ }^{13}$

La correlación entre la práctica del colecho y los resultados de la lactancia ha sido demostrada en numerosos estudios epidemiológicos ${ }^{14} \mathrm{y}$ confirmada por las madres en estudios cualitativos. ${ }^{15,16}$ La lactancia materna tiene una función protectora con respecto a la muerte súbita del lactante (MSL) ${ }^{17-19}$ y es mayor cuando la lactancia es exclusiva. ${ }^{20}$

Algunas publicaciones muestran una asociación entre colecho y la MSL. ${ }^{21,22}$ Sin embargo, un estudio realizado en el Reino Unido en el año 2014, en el cual se incluyeron 400 casos de síndrome de muerte súbita del lactante (SMSL) y 1400 controles, no encontró una asociación entre el colecho y la MSL en ausencia de los siguientes factores de riesgo: consumo materno de alcohol, drogas y cigarrillo; prematuros / bajo peso y cuando alguno de los progenitores dormían junto al bebé en una silla, sillón, hamaca mecedora, sofá, etc. ${ }^{23}$ La controversia complejiza las indicaciones del pediatra. ${ }^{24}$

Esta recomendación propone ofrecer a los padres una amplia y balanceada comunicación, que incluya tanto los beneficios como los riesgos del colecho, para permitirles adoptar una decisión informada, como sugiere el Instituto Nacional de Salud y Atención de Excelencia del Reino Unido (National Institute for Health and Care Excellence, NICE), 2014. ${ }^{25}$

\section{Conceptos generales}

El SMSL se refiere a la muerte durante el sueño de un lactante aparentemente sano, sin otra causa demostrable. ${ }^{26}$ En nuestro país, hablamos de muerte súbita e inesperada del lactante, ya que no se realizan ni autopsia por un patólogo pediatra ni estudio de la escena de la muerte que permitan llegar a un diagnóstico de certeza. Adquiere relevancia, entonces, poder distinguir las muertes por asfixia, que serían evitables. ${ }^{27}$

Los profesionales de la salud nos sentimos abrumados cuando dialogamos con los padres acerca del colecho y la MSL. ${ }^{25}$ Por este motivo, los integrantes de la Subcomisión de Lactancia Materna de la Sociedad Argentina de Pediatría y del Grupo de Trabajo en Muerte Súbita e Inesperada del Lactante hemos elaborado esta recomendación en forma conjunta para ayudar al equipo de salud en el diálogo con los padres, de manera que los riesgos sean identificados y disminuidos, en lugar de intentar promocionar prácticas restrictivas que no siempre pueden ser aplicadas por los padres en la vida cotidiana.

La conversación con los padres resulta compleja y se generan controversias, y el contenido de nuestro mensaje, a veces, resulta disfuncional o poco realista en relación con las realidades que viven las familias, debido a que la práctica del colecho conlleva factores culturales, sociales y económicos. ${ }^{11,28}$

El mensaje brindado a los padres con la consigna terminante de que nunca realicen colecho con sus hijos no contribuye a disminuir el riesgo de MSL, en virtud de lo siguiente:

- Los niños pequeños se despiertan frecuentemente durante la noche y demandan ser alimentados y atendidos en algún lugar. ${ }^{29}$ Las madres eligen, entonces, darles de mamar a sus hijos en un sillón, situación de extremo riesgo, ${ }^{30,31}$ atentas al consejo de "nunca colecho".

- Las madres intentan incorporarse y sentarse en la cama en vez de permanecer acostadas a la hora de darles de mamar a sus hijos. Como los niños se alimentan con gran frecuencia durante la noche, las madres podrían quedarse dormidas en una posición que es mucho más peligrosa que la de estar acostadas. ${ }^{16}$

- Si bien algunos niños permanecen calmos en sus cunas en los períodos en los cuales no se alimentan, otros lloran de manera inconsolable. Algunos padres adhieren, entonces, a la norma de la psicología conductista del Duérmete, niño, que propone un método de adiestramiento para enseñar a los niños a dormir solos, que incluye dejar al niño llorando en su cuna. Esta práctica genera una situación de estrés para la díada. ${ }^{32-34}$

- Muchas familias manifiestan que, pese a la prescripción de "nunca colecho", transgreden la recomendación y duermen con sus hijos. ${ }^{16}$ Resulta imposible eliminar absolutamente el riesgo de MSL. Sin embargo, mediante una conversación empática con los padres, podemos, potencialmente, disminuir las muertes por colecho en un $90 \% .^{35}$ 
El riesgo no es inherente al colecho en sí, sino a las circunstancias en las que ocurre. ${ }^{23,27} \mathrm{Lo}$ que adiciona factores de riesgo son las prácticas de sueño inseguro: exceso de abrigo, ${ }^{36}$ dormir en posición boca abajo o de costado, ${ }^{37}$ presencia de almohadas, almohadones, acolchados, mantas, colchón blando, ${ }^{38,39}$ dejar al niño en una cama sin supervisión de adultos, ${ }^{40,41}$ alimentarlo solo con fórmula, ${ }^{42}$ usar nidos de contención ${ }^{43}$ o el huevito para el auto en la cama de los padres. ${ }^{44}$

Es contundente la evidencia científica que muestra que el colecho aumenta significativamente el riesgo de MSL en aquellos casos en que los padres fuman o la madre fumó durante el embarazo, ${ }^{45,46}$ ingieren alcohol, drogas psicoactivas recreativas o sedantes, ${ }^{23,27}$ en especial si el niño es prematuro o sufrió una restricción del crecimiento intrauterino. ${ }^{47}$ Sin embargo, hay otros niños que pueden ser vulnerables - por alteraciones genéticas en los neurotransmisores-, a los que no podemos identificar en la actualidad. ${ }^{48}$

Cuando el recién nacido está en contacto piel a piel con su madre, puede estar en posición prona sobre el torso materno. En estas circunstancias, se debe cuidar que la cabeza del bebé esté girada hacia un lado con la nariz y la boca desobstruidas y con el cuello no flexionado. La madre debe permanecer despierta y alerta para evitar obstrucciones de las vías aéreas ${ }^{49}$ y caídas. ${ }^{50}$

El colecho no debería ser la única opción posible para familias carenciadas, sino una elección luego de conocer los beneficios y los riesgos. En los casos en los que el colecho es consecuencia de condiciones de vulnerabilidad y/o hacinamiento, las familias deben estar informadas y se les debe garantizar un programa integral de promoción de la lactancia, de sueño seguro y ofrecerles una cuna para su niño. ${ }^{51}$

No podemos "prohibir" el colecho, pero tampoco podemos dejar de advertir a las familias los potenciales riesgos. ${ }^{25}$ Cada familia debe elegir con libertad y conocimiento, por lo cual es imprescindible dar información completa a los padres, tanto de los beneficios de la práctica de colecho como de las condiciones que aumentan el peligro durante esta práctica, más que promover restricciones difíciles de cumplir en la vida cotidiana. ${ }^{52}$

La conversación con los padres debe proporcionar información comprensible, basada en sus necesidades. En definitiva, son los padres quienes, con todo el cuerpo de evidencia disponible, tomarán la decisión.

\section{Consideraciones relevantes}

- El lugar más seguro para que duerma el bebé es en una cuna al lado de la cama de sus padres. ${ }^{1-3,53}$

- La práctica que impone que los niños duerman solos los primeros meses de vida, en una habitación distinta a la de los padres, tiene una vigencia de poco más de un siglo, se lleva a cabo en muy pocos países del mundo y aumenta el riesgo de MSL. 54,55

- Todos los padres deben recibir el mensaje de que la cohabitación durante los primeros seis meses de la vida disminuye el riesgo de MSL, independientemente de la alimentación que reciba el niño. ${ }^{53}$

- Las evidencias actuales muestran que el colecho presenta pocos beneficios para aquellos niños que se alimentan con fórmula exclusiva. ${ }^{56}$

- Las familias deben ser informadas sobre la importancia de la lactancia materna como un factor protector relevante para disminuir la MSL. ${ }^{19}$

- No se observó incremento del riesgo cuando el niño compartía la cama de la madre por períodos cortos con el propósito de amamantar e incrementar el contacto piel a piel..$^{57,58}$

- Las muertes en situación de colecho se asocian, al menos, con un factor de riesgo para la MSL: posición boca abajo, alimentación exclusiva con fórmula, compartir la cama con otro niño, acostar al niño sobre una almohada. ${ }^{59}$

- Dormir con un niño en un sofá, sillón o silla mecedora aumenta el riesgo considerablemente comparado con dormir en otras superficies (por ej., una cuna)., ${ }^{1,31}$

- El NICE concluye que no existe, en la actualidad, suficiente evidencia como para prohibir el colecho de manera absoluta cuando no hay factores de riesgo. ${ }^{25}$

- Los padres tienen el derecho a saber tanto los riesgos del colecho como sus beneficios conocidos en la actualidad, sobre la base de la mejor información científica publicada. ${ }^{60}$

Para que los padres puedan tomar una decisión informada en relación con el ambiente seguro para el sueño de sus hijos, se les deben proporcionar las pautas que disminuyen el riesgo de MSL y lesiones en relación con la práctica del colecho, ${ }^{61}$ que figuran en el Apéndice A. Cada familia debe poder tener una cuna para su hijo y recibir información sobre sueño seguro ${ }^{62}$ (véase el Apéndice B). 


\section{Recomendación sobre colecho en el hogar}

El colecho es especialmente peligroso en las siguientes circunstancias y se debe evitar siempre:

- Cuando el padre y / o la madre fuman, aunque no lo hagan en la cama (OR 2,3-21,6). 1,45,57,62

- Cuando la madre fumó durante el embarazo. $1,45,46,57$

- Cuando el niño/a es prematuro o presentó restricción del crecimiento intrauterino. ${ }^{1}$

- Situaciones de colecho con múltiples personas (OR 5,4). ${ }^{38}$

- Cuando alguno de los padres consume alcohol $^{14,27,53}$ y / o drogas ilícitas (OR 1,66$89,7) .42$

- Cuando el colecho se realiza con alguna persona que no sea su padre o madre (OR 5,4). ${ }^{38}$

- Cuando existen almohadas y/o ropa de cama mullida en el lecho donde duerme el bebé junto a sus padres (OR 2,8-4,1).27,39

- Cuando el niño/a queda durmiendo solo en la cama de un adulto. ${ }^{41}$

\section{Consejo personalizado}

Los siguientes grupos vulnerables requieren un mensaje personalizado para asegurarnos de que ha sido incorporado y comprendido, dado que, en estos casos, podría haber un riesgo aumentado para MSL:

- Familias de bajo nivel social y/o económico.

- Madres jóvenes y/o adolescentes con más de un hijo.

- Familias con hijos prematuros y/o de bajo peso.

- Padres que ingieren drogas ilícitas, drogas sedantes o alcohol.

- Padres fumadores.

- Situación de hacinamiento habitacional. ${ }^{1}$

\section{CONCLUSIÓN FINAL}

Si bien la cohabitación sin colecho es el escenario más seguro, hay también una realidad y es que el colecho, además de ser una práctica muy frecuente en todos los estratos sociales, tiene una asociación positiva con la lactancia materna y esta constituye un factor de protección para la MSL.

Es necesario dejar a los padres decidir sobre la base de sus convicciones, explicándoles detalladamente los beneficios y los escenarios de riesgo para la práctica del colecho. Se contraindica formalmente la práctica del colecho ante la presencia de factores de riesgo, como la prematurez, el consumo de drogas sedantes, ilícitas o alcohol, el tabaquismo y la alimentación con fórmula exclusiva.

\section{APÉNDICE A}

Sugerencias para disminuir la morbimortalidad asociada al colecho

- No practicar colecho si alguno de los padres presenta una disminución de la capacidad para despertarse debido a fatiga extrema, ingesta de medicación sedante, alcohol o drogas ilícitas.

- Si el bebé duerme en la cama con los padres, no debe hacerlo sobre una superficie blanda (por ejemplo, almohada, colchón de agua, colchas mullidas, piel de cordero).

- Ubicar al bebé al costado de uno de los padres, no en el medio de ambos.

- El colecho con hermanos, hermanas u otros familiares es desaconsejable.

- El colecho debe desaconsejarse cuando conviven muchas personas en la misma habitación.

- Conocer los potenciales peligros asociados con esta práctica:

- Aplastamiento por parte de un adulto.

- Que el bebé quede "atrapado" entre el colchón y la pared o entre el colchón y el respaldo de la cama.

- Que el bebé quede con su cabeza boca abajo en algún hueco del colchón.

- Caídas del bebé de la cama al suelo.

- Extremar los cuidados en los niños/as con afecciones neurológicas y con restricción de sus movimientos.

- No practicar el colecho si alguno de los padres fuma.

- No practicar el colecho en un sillón o sofá.

\section{APÉNDICE B}

Recomendaciones para un sueño seguro

durante el primer año de vida

- Promover la lactancia materna exclusiva hasta los 6 meses.

- Dormir boca arriba.

- Compartir la habitación sin compartir la cama, como escenario más seguro.

- El colchón debe ser firme y del mismo tamaño de la cuna.

- No colocar ningún tipo de objeto dentro de la cuna (almohada, nido, rollo, chichonera, edredones, colchas, frazadas gruesas o juguetes).

- Tapar al niño hasta las axilas con los brazos por fuera de la ropa de cama y sujetar con firmeza la ropa de cama.

- Evitar el exceso de abrigo.

- Temperatura ambiente moderada. 
- No prohibir el chupete para dormir, cuando la lactancia esté bien establecida.

- Realizar los controles periódicos durante el embarazo.

- No fumar durante el embarazo.

- No exponer al niño al humo del tabaco.

- Utilizar la silla de seguridad para el auto (huevito) exclusivamente para viajar en un vehículo.

- Vacunación completa.

- Cuna clásica que cumpla con las especificaciones del "Consenso sobre mobiliario infantil" de la Sociedad Argentina de Pediatría. ${ }^{63}$

\section{REFERENCIAS}

1. Blair PS, Fleming PJ, Smith IJ, et al. Babies sleeping with parents: case-control study of factors influencing the risk of the sudden infant death syndrome. CESDI SUDI Research Group. BMJ 1999;319(7223):1457-61.

2. Mitchell EA, Thompson JMD. Co-sleeping increases, the risk of SIDS, but sleeping in the parents' bedroom lowers it. In: Rognum TO, edit. Sudden Infant Death Syndrome: New Trends in the Nineties. Oslo, Norway: Scandinavian University Press; 1995:266-9.

3. Tappin D, Ecob R, Brooke H. Bedsharing, roomsharing, and sudden infant death syndrome in Scotland: a casecontrol study. J Pediatr 2005;147(1):32-7.

4. La Biblia. Libro I de los Reyes. 3.16-28.

5. Russell-Jones DL. Sudden infant death in history and literature. Arch Dis Child 1985;60(3):278-81.

6. Nelson EA, Schiefenhoevel W, Haimerl F. Child care practices in nonindustrialized societies. Pediatrics 2000; 105(6): E75.

7. Blair PS, Heron J, Fleming PJ. Relationship between bed sharing and breastfeeding longitudinal, population-based analysis. Pediatrics 2010;126(5): e119-26.

8. Horsley T, Clifford T, Barrowman N, etal. Benefits and harms associated with the practice of bed sharing: a systematic review. Arch Pediatr Adolesc Med 2007;161(3):237-45.

9. Huang Y,Hauck FR, Signore C, et al. Influence of bedsharing activity on breastfeeding duration among US mothers. JAMA Pediatr 2013:167(11):1038-44.

10. Mckenna JJ, Mosko SS, Richard CA. Bedsharing promotes breastfeeding. Pediatrics 1997;100 (2 Pt 1):214-9.

11. Ball HL. Breastfeeding, bed sharing and infant sleep. Birth 2003:30(3):181-8

12. Babies sharing their mothers ' bed while in hospital: a sample policy. UNICEF UK Baby Friendly Initiative, May 2004. [Acceso: 24 de mayo de 2017]. Disponible en: http: / / www.nhshighland.scot.nhs.uk/YourHealth/Documents/ Breastfeeding/UNICEF Sample Policy - Babies Sharing their Mothers Bed while in Hospital.pdf.

13. Jenik A. Colechoy Síndrome de MuerteSúbita del Lactante: una relación conflictiva. Arch Argent Pediatr 2001;99(3): 228-32.

14. Santos IS, Mota DM, Matijasevich A, et al. Bed-Sharing at 3 Months and Breast-Feeding at 1 Year in Southern Brazil. J Pediatr 2009;155(4):505-9.

15. Joyner BL, Oden RP, Ajao TI, et al. Where should my baby sleep: a qualitative study of African American infant sleep location decisions. J Natl Med Assoc 2010;102(10):881-9.

16. Kendall-TackettK, Cong Z, Hale TW. Mother-InfantSleep Locations and Nighttime Feeding Behavior: U.S. Data from the Survey of Mothers' Sleep and Fatigue. Clinical Lactation 2010;1:27-31.

17. Shamberger R. Breast feeding Associated with Reduced Sudden Infant Syndrome and Infant Mortality. Med J Obstet Gynecol 2014:2(1):1021.

18. Ip S, Chung M, Raman G, et al. A summary of the Agency for Healthcare Research and Quality's evidence report on breastfeeding in developed countries. Breastfeed Med 2009;4(Suppl 1):S17-30.

19. Hauck FR, Thompson JM, Tanabe KO, et al. Breastfeeding and reduced risk of sudden infant death syndrome: a metaanalysis. Pediatrics 2011;128(1):103-10.

20. Vennemann MM, Bajanowski T, Brinkmann B, et al. Does breastfeeding reduce the risk of sudden infant death syndrome? Pediatrics 2009;123(3): e406-10.

21. Mitchell EA, Taylor BJ, Ford RPK, et al. Four modificale and another major risk factors for cot death: the New Zeland study. J Paediatr Child Health 1992;28(Suppl 1):S3-8.

22. Ruys JH, de Jonge GA, Brand R, et al. Bed-sharing in the first four months of life: a risk factor for sudden infant death. Acta Paediatr 2007;96(10):1399-403.

23. Blair PS, Sidebotham P, Pease A, et al. Bed-sharing in the absence of hazardous circumstances: is there a risk of sudden infant death syndrome? An analysis from two case-control studies conducted in the UK. PLoS One 2014;9(9):e107799.

24. Morgan KH, Groer MW, Smith LJ. The Controversy About What Constitutes Safe and Nurturant Infant Sleep Environments. J Obstet Gynecol Neonatal Nurs 2006; 35(6):684-91.

25. NICE. Posnatal care up to 8 weeks after birth. 2006. [Acceso: 24 de mayo de 2017]. Disponible en: https:/ / www.nice. org.uk/guidance / cg37 / resources / postnatal-care-up-to8-weeks-after-birth-pdf-975391596997.

26. Jenik A, Grad E, Orazi V, et al. Consideraciones sobre el sueño seguro del lactante. Resumen ejecutivo. Arch Argent Pediatr 2015;113(3):285-7.

27. Blair PS, Sidebotham P, Evason-CoombeC, etal.Hazardous cosleeping environments and risk factors amenable to change: case-control study of SIDS in south west England. BMJ 2009;339:b3666.

28. Ball HL. Reasons to bed-share: why parents sleep with their infants. J Reprod Infant Psychol 2002;20(4): 207-21.

29. Kent JC, Mitoulas LR, Cregan MD, et al. Volume and Frequency of Breastfeeding and Fat Content of Breast Milk Throughout the Day. Pediatrics 2006;117(3): e387-95.

30. Rechtman LR, Colvin JD, Blair PS, et al. Sofas and infant mortality. Pediatrics 2014;134(5):e1293-300.

31. Byard RW, Beal S, Blackbourne B, et al. Specific dangers associated with infants sleeping on sofas. J Paediatr Child Health 2001;37(5):476-8.

32. Gunnar MR, Donzella B. Social regulation of the cortisol levelsinearlyhumandevelopment.Psychoneuroendocrinology 2002;27(1-2):199-220.

33. Bell SM, Ainsworth MD. Infant crying and maternal responsiveness. Child Dev 1972;43(4):1171-90.

34. St. James-Roberts I. Infant crying and sleeping: helping parents to prevent and manage problems. Sleep Med Clin 2007;2(3):363-75.

35. Co-sleeping and SIDS: A guide for health professionals. UNICEF, 2016. [Acceso: 24 de mayo de 2017]. Disponible en: http: / / lrsb.org.uk/ uploads / co-sleeping-sids---aguide-for-professionals.pdf.

36. Tuffenell CS, Petersen SA, Wailoo MO. Higher rectal temperatures in co-sleeping infants. Arch Dis Child 1996;75(3):249-50.

37. Gessner BD, Ives GC, Perham-Hester KA. Association between sudden infant death syndrome and prone sleep position, bed sharing, and sleeping outside an infant crib 
in Alaska. Pediatrics 2001;108(4):923-7.

38. Hauck FR, Herman SM, Donovan M, et al. Sleep environment and the risk of sudden infant death syndrome in an urban population: the Chicago Infant Mortality Study. Pediatrics 2003;111(5 Pt 2):1207-14.

39. FuLY, Moon RY,HauckFR. Bed sharing among blackinfants and sudden infant death syndrome: interactions with other known risk factors. Acad Pediatr 2010;10(6):376-38.

40. Buckley SJ. Ten tips for safe sleeping. 2010. [Acceso: 24 de mayo de 2017]. Disponible en: http:/ / sarahbuckley.com/ ten-tips-for-safe-sleeping.

41. McKenna JJ, McDade T. Why babies should never sleep alone: a review of the co-sleeping controversy in relation to SIDS, bedsharing and breast feeding. Paediatr Respir Rev 2005;6(2):134-52.

42. Carpenter R, McGarvey C, Mitchell EA, et al. Bed sharing when parents do not smoke: is there a risk of SIDS? An individual level analysis of five major case-control studies. BMJ Open 2013;3(5):e002299.

43. Rossato NE. Nidos de contención para recién nacidos y riesgo de muerte súbita del lactante. Arch Argent Pediatr 2013;111(1):62-8.

44. Côté A, Bairam A, Deschenes M, et al.Sudden infant deaths in sitting devices. Arch Dis Child 2008;93(5):384-9.

45. Scragg R, MitchellEA, Taylor BJ, et al. Bed sharing, smoking, and alcohol in the sudden infant death syndrome. New Zealand CotDeath Study Group. BMJ1993;307(6915):1312-8.

46. Vennemann MM, Hense HW, Bajanowski T, et al. Bed sharing and the risk of sudden infant death syndrome: can we resolve the debate? J Pediatr 2012;160(1):44-8.e2.

47. Blair PS, Platt MW, Smith IJ, et al. Sudden infant death syndrome and sleeping position in pre-term and low birth weight infants: an opportunity for targeted intervention. Arch Dis Child 2006;91(2):101-6.

48. Bergman NJ. Proposal for mechanisms of protection of supine sleep against infant death syndrome: an integrated mechanism review. Pediatr Res 2015;77(1-1):10-9.

49. Feldman K, Whyte RK. Two cases of apparent suffocation of newborns during side-lying breastfeeding. Nurs Womens Health 2013;17(4):337-41.

50. Heafner L, Suda D, Casalenuovo N, et al. Development of a tool to assess risk for falls in women in hospital obstetric units. Nurs Womens Health 2013;17(2):98-107.

51. Hauck FR, Tanabe KO, McMurry T, et al. Evaluation of bedtime basics for babies: a national crib distribution program to reduce the risk of sleep-related sudden infant deaths. J Community Health 2015;40(3):457-63.

52. Mitchell EA. Bed sharing and the risk of sudden infant death: parents need clear information. Curr Pediatr Rev 2010;6(1):63-6.

53. Task Force on Sudden Infant Death Syndrome. SIDS and Other Sleep-Related Infant Deaths: Updated 2016 Recommendations for a Safe Infant Sleeping Environment. Pediatrics 2016;138(5):e20162938.

54. Carpenter RG, Irgens LM, Blair PS, et al. Sudden unexplained infant death in 20 regions in Europe: case control study. Lancet 2004;363(9404):185-91.

55. Scragg RKR, Mitchell EA, Stewart AW, et al. Infant roomsharing and prone sleep position in sudden infant death syndrome. Lancet 1996;347(8993):7-12.

56. McKennaJ.Safe Cosleeping Guidelines. University of Notre Dame, 2012. [Acceso: 27 de enero de 2012]. Disponible en: http: / / cosleeping.nd.edu/ safe-co-sleeping-guidelines / .

57. Fleming PJ, Blair PS, Bacon C, et al. Environment of infants during sleep and risk ofthe sudden infant death syndrome: results of 1993-5 case-control study for confi dential inquiry into stillbirths and deaths in infancy. Confidential Enquiry into Stillbirths and Deaths Regional Coordinators and Researchers. BMJ 1996;313(7051):191-5.

58. McGarvey C, McDonnell M, Chong A, et al. Factors relating to the infant's last sleep environment in sudden infant death syndrome in the Republic of Ireland. Arch Dis Child 2003;88(12):1058-64.

59. Blabey MH, Gessner BD. Infant bed-sharing practices and associated risk factors among births and infant deaths in Alaska. Public Health Rep 2009;124(4):527-34.

60. Ball HL. Bed-sharing and co-sleeping: research overview. New Digest 2009;48:22-7.

61. Australian College of Midwives Position Statement on bed-sharing and Co-sleeping. 2014. [Acceso: 24 de mayo de 2017]. Disponible en: https: / / 7-midwives.cdn.aspedia. net / sites / default / files / uploaded-content / field_f_ content_file/acm_position_statement_bedsharing_cosleeping_20141024.pdf.

62. Mitchell EA, Cowan S, Tipene-Leach D. The recent fall in postperinatal mortality in New Zealand and the Safe Sleep Programme. Acta Paediatr 2016;105(11):1312-20.

63. Comité Nacional de Prevenciones y Lesiones. Consenso sobre mobiliario infantil seguro. Sociedad Argentina de Pediatría, 2016. [Acceso:24 de mayo de 2017]. Disponible en:www.sap.org.ar/uploads/consensos/consenso-sobremobiliario-infantil-seguro-nbsp2016.pdf. 